OPEN ACCESS

Edited by:

Jordi Anton,

Hospital Sant Joan de Déu

Barcelona, Spain

Reviewed by:

Ozgur Kasapcopur:

Istanbul University-Cerrahpasa, Turkey David Piskin,

Lawson Health Research Institute, Canada Itziar Alonso,

Pediatric Research Hospital Sant Joan de Déu, Spain

${ }^{*}$ Correspondence: Maria Cristina Maggio mariacristina.maggio@unipa.it

Specialty section: This article was submitted to

Pediatric Rheumatology,

a section of the journal

Frontiers in Pediatrics

Received: 09 July 2020 Accepted: 12 November 2020 Published: 07 December 2020

Citation:

Maggio MC, Corsello G, Salvo G,

Puma DG and Cimaz R (2020)

Brainstem Auditory Evoked Potentials and Visual Potentials in Kawasak

Disease: An Observational

Monocentric Study

Front. Pediatr. 8:581780.

doi: 10.3389/fped.2020.581780

\section{Brainstem Auditory Evoked Potentials and Visual Potentials in Kawasaki Disease: An Observational Monocentric Study}

\author{
Maria Cristina Maggio ${ }^{1 *}$, Giovanni Corsello ${ }^{1}$, Giuseppe Salvo ${ }^{1}$, \\ Domenico Giuseppe Puma ${ }^{2}$ and Rolando Cimaz ${ }^{3}$ \\ 1 University Department PROMISE of Health Promotion Sciences Maternal and Infantile Care, Internal Medicine and Medical \\ Specialities "G. D'Alessandro", University of Palermo, Palermo, Italy, ${ }^{2}$ Pediatric Neuropsychiatry Operative Unit, Children \\ Hospital "G. Di Cristina", ARNAS, Palermo, Italy, ${ }^{3}$ Department of Clinical Sciences and Community Health, University of \\ Milan, Milan, Italy
}

Background: Kawasaki Disease is a systemic vasculitis, particularly involving coronary arteries. Rare involvement of other vascular districts is described, as central nervous system arteries, leading to a vasculitic neuropathy. Sensorineural hearing loss and alterations of evoked potentials are uncommonly reported complications.

Methods: In an observational monocentric study, 59 children (37 males; 22 females; mean age: $2.7 \pm 2.2$ years) with documented Kawasaki Disease were enrolled. No risk factors for hearing loss and/or neurological impairment were identified in the cohort. Brainstem auditory evoked potentials and visual evoked potentials were correlated with clinical, hamatological and radiological data, evaluated in the acute phase of the Kawasaki Disease, and during the follow-up.

Results: Evoked potentials were altered in 39/59 patients (66\%): of these, 27/39 (69\%) showed altered IV and V waves and/or III-V interwave latencies of brainstem auditory evoked potentials; 4/39 (10\%) showed pathological visual evoked potentials; $8 / 39(21 \%)$ had abnormalities of both brainstem auditory evoked potentials and visual evoked potentials. No permanent deafness was reported.

Conclusion: Abnormalities in visual evoked potentials were not significantly correlated with coronary artery lesions; however, the presence of abnormalities of brainstem auditory evoked potentials were associated with the risk of coronary artery lesions.

Keywords: kawasaki disease, brainstem auditory evoked potentials, visual evoked potentials, coronary artery lesions, intravenous immunoglobulins

\section{INTRODUCTION}

Coronary artery lesions (CAL) and/or cardiac lesions are the fearsome evolution of Kawasaki disease (KD). Nevertheless, other vascular districts may be involved less frequently, such as central nervous system (CNS) arteries $(1,2)$, peripheral vasa nervorum and perineural blood vessels, leading to a vasculitic neuropathy of acoustic nerve and optic nerve $(3,4)$. 
A severe cochlear or vessel wall inflammation can induce hearing loss and/or subclinical alteration of brainstem auditory evoked potentials (BAEPs). Optic nerve could be involved as well, with a pathological pattern of visual evoked potentials (VEPs). However. these abnormalities are sporadic and have been described just in a few reports $(4,5)$.

In the literature, only few studies focused on BAEPs and VEPs in $\mathrm{KD}$ (6). There are also some case reports of auditory loss secondary to $\operatorname{KD}(4,5)$.

Sensorineural hearing loss may be underreported, since a reduced hearing acuity may be only transient and since when the condition is present in young children it could not be promptly recognized. Hearing loss is often detected only by audiometry or, in young children in whom tone audiometry is difficult to be carried out, by BAEPs.

Some reports showed that up a third of KD children had a sensorineural auditory loss during the acute and subacute phases of the disease, and that the auditory loss could be maintained even six months after diagnosis $(7,8)$. The authors observed a positive association of this complication with anemia and thrombocytosis. VEPs and BAEPs may be pathological in the acute phase of $\mathrm{KD}$, however they can normalize during the follow-up. This finding may be the sign of systemic vasculitis, involving CNS.

CAL may have poor correlation with clinical signs; however, hematological parameters, expression of critical cytokine secretion (such as leucocytosis, increased neutrophil percentage, elevation of CRP, increased transaminases, hypoalbuminemia and hyponatremia), are correlated with a more severe evolution and an increased risk to develop CAL. Pharmacological approach of KD with IVIG within 7-10 days since KD onset is efficacious to prevent CAL. However, there are no data about hematological parameters and the treatment choice on the prevention of evoked potentials alterations.

A systemic vasculitis could involve many districts at the same time and in the acute phase of the disease; the synchronous involvement of coronaries and CNS districts in KD has not yet been demonstrated.

\section{Aims of the Study}

Aims of the study were:

- the evaluation of VEPs and BAEPs in children affected by KD, in the acute phase of the disease and during the follow-up;

- the evaluation of the role of evoked potentials as an added tool to detect occurrence of vasculitis in the anatomic and functional systems respectively explored by VEPs and BAEPs;

- the correlation of VEPs and BAEPs with clinical signs, hematological parameters, treatment with IVIG, aspirin and other drugs;

- the correlation between CAL and/or cardiac lesions, and dysfunction of VEPs and BAEPs at diagnosis and during the follow up.

Abbreviations: KD, Kawasaki Disease; BAEPs, brainstem auditory evoked potentials; VEPs, visual evoked potentials; CAL, coronary artery lesions; CRP, C-reactive protein; ESR, Erythrocyte sedimentation rate.

\section{MATERIALS AND METHODS}

\section{Patients}

In an observational monocentric study, we enrolled 59 children $(37 \mathrm{M}$; $22 \mathrm{~F}$; mean age: $2.7 \pm 2.2$ years; range 3 months -10 years) with documented KD. All the patients, followed from 2012 to 2018 in the Pediatric Clinic of Palermo, Children Hospital "G. Di Cristina," ARNAS Palermo, with a good compliance to the procedures of evaluation of evoked potentials, and no risk factors for hearing loss and/or neurological impairment (CNS congenital diseases, developmental delay, ASA intoxication, etc.) were included. Of those, 37 children (63\%) had typical KD, eight patients $(14 \%)$ atypical $\mathrm{KD}$, and 14 patients (23\%) an incomplete form. All patients were treated with $2 \mathrm{~g} / \mathrm{kg}$ of intravenous immunoglobulins (IVIG) and acetylsalicylic acid (ASA) at the dosage of $30-50 \mathrm{mg} / \mathrm{kg} /$ day in the acute phase, and $3-5 \mathrm{mg} / \mathrm{kg}$ /day as antiplatelet prophylaxis. BAEPs and VEPs were correlated with clinical, hematological and radiological data, evaluated in the acute phase of the KD, and during the follow up.

\section{Clinical and Demographic Data}

Age, gender, days of illness at initial treatment and response to IVIG and ASA; rash; changes of lips and oral mucosa; conjunctivitis; cervical lymphadenopathy.

\section{Hematological Parameters}

White blood cell count, neutrophils percentage, platelet count, hemoglobin, C-reactive protein (CRP), erythrocyte sedimentation rate (ESR), transaminases, gamma-glutamyl transferase, ferritin, albumin, sodium, D-dimer.

\section{Imaging Data}

Chest x-ray, echocardiogram, abdominal ultrasound.

\section{Diagnostic Criteria}

KD was diagnosed following the internationally approved criteria $(9,10)$ :

A. Typical KD was diagnosed when fever lasting more than 5 days was associated with $\geq 4$ clinical criteria:

1. bilateral non-exudative conjunctivitis

2. changes of oral mucosa and lips

3. changes of the extremities

4. polymorphous exanthema

5. cervical lymphadenopathy.

B. Incomplete $\mathrm{KD}$ was suggested in every infant showing fever lasting more than 5 days with documented systemic inflammation if $<4$ main clinical features were found after exclusion of many febrile illnesses.

C. Atypical $\mathrm{KD}$ was diagnosed if fever lasting more than 5 days, not otherwise explained, was associated with nonclassic manifestations (as sensorineural hearing loss, aseptic meningitis, seizures, peripheral facial nerve palsy, acute abdomen, pancreatitis, gallbladder hydrops, pneumonia, arthritis, orchitis, renal impairmant, sterile pyuria). CAL documented by echocardiography confirmed the diagnosis. 


\section{Ethics and Ethics Committee}

Ethics committee approval according to local regulations was not necessary since this was part of our clinical practice and since deidentified data were used for statistical analysis. However, the study involving human participants was reviewed and approved by the ethics committee Palermo 1 (ARNAS Civico, Palermo, Italy). The patients' legal guardian provided written informed consent to participate in this study. The written consent is in the documents in the hospital "G. Di Cristina", ARNAS Palermo, Italy.

\section{Methods}

VEPs and BAEPs were recorded by a MYOQUICK SystemPLUS Evolution (Micromed, Italian). The relevant recording techniques are described hereunder.

The latencies evaluation of waves I, III and V were performed in all patients together with the amplitudes of waves I and V. Furthermore, the V/I amplitude ratio, and the I-III and III$\mathrm{V}$ interwave latencies were calculated. Recorded abnormalities consisted of decreased amplitude of wave $\mathrm{V}$ and prolonged III- $\mathrm{V}$ interpeak latencies.

The recordings were collected in the acute phase of the disease, in the few days $(1-7,9-11)$ after defervescence occurred, before discharge from the hospital, and during follow-up (6 and 12 months after discharge).

\section{VEPs}

VEPs were recorded from an active midline occipital electrode over the visual cortex at $\mathrm{O}_{\mathrm{z}}$, with a midline frontal reference electrode at $\mathrm{F}_{\mathrm{z}}$, according to the Recommendations and Guidelines of the International Federation of Clinical Neurophysiology (IFCN) Committee (12) and International Society for Clinical Electrophysiology of Vision (ISCEV) (13). On the basis of children's age and of his/her degree of cooperation during the exam, different types of stimulus have been used.

Flash VEPs were performed with monocular stimulation. The unstimulated eye was occluded to avoid extraneous and unwanted stimulation. The potentials were carried out using a flash (brief luminance increment) with a stimulation force of $3 \mathrm{~cd} \mathrm{~s} \mathrm{~m}^{-2}$ (photopic candles seconds per square meter) which subtends a visual field of at least $20^{\circ}$ in a dimly illuminated room.

\section{- Pattern Reversal Visual Evoked Potentials (PRVEPs) were performed with monocular stimulation and full-field stimulation.}

The standard stimulus for VEPs is a checkerboard model in which the squares take turns from black to white: the dark squares become light and vice versa without changing the general luminance of the display.

The pattern was reversed 100 times at $1 \mathrm{~Hz}$ and the results were then averaged; A repeated trial of averaged stimuli was also recorded.

\section{BAEPs}

The BAEPS were carried out and interpreted according to the guidelines of the American Clinical Neurophysiology Society (ACNS) (14).
BAEPs were recorded from an active electrode positioned over $\mathrm{Cz}$ and referred to the right (M2) and to the left (M1) mastoid process. Waveforms are recorded from ipsilateral and contralateral pathways simultaneously, allowing easier recognition of individual peaks.

The following regards others technical details:

- the stimuli consisted of $100 /$ microseconds clicks delivered to each ear in turn through pre-calibrated shielded earphones at a rate of $10.6 \mathrm{~Hz}$.

- the stimulus intensity was $60 \mathrm{~dB}$ above the hearing threshold (14) for each individual ear with the contralateral ear masked by white noise of $40 \mathrm{~dB}$ below the stimulus intensity;

- Thousand and five hundred or more stimuli, depending on the shape and amplitude of the potentials, were averaged;

- the latencies of waves I, III, and V were measured together with the amplitudes of waves I and V. The V/I amplitude ratio, and the I-III and III-V interweaves latencies were also calculated.

\section{Statistics}

Patients were classified by gender, age, treatment duration and evoked potentials normal or altered. Correlation between the analyzed parameters collected were performed using Chi-square test. All variables were tested for normal distribution using the Anderson-Darling normality test. All ordinal data were expressed as numbers and percentages. Calculations were done using "MiniTAB release 13.1 Statistical Software."

\section{RESULTS}

\section{Evoked Potentials Abnormalities}

The data of evoked potentials findings for all the patients, divided in three groups of KD (typical, atypical, incomplete), are reported in Table 1.

Evoked potentials were altered in 39/59 patients (66\%) (26 M; $13 \mathrm{~F})$ : among these, $27 / 39$ (69\%) (18 M; $9 \mathrm{~F})$ had pathological BAEPs; 4/39 (10\%) (2 M; 2 F) had pathological VEPs; 8/39 (21\%) $(6 \mathrm{M} ; 2 \mathrm{~F})$ had abnormalities of both BAEPs and VEPs. Hence, BAEPs were altered in 35 patients (24 M; $11 \mathrm{~F}$ ) (Table 2).

Pathological BAEPs waves were documented as bilateral in most of the patients. However, 12 patients (31\%) showed unilateral pathological BAEPs.

In two patients hearing loss was documented, and one of them showed neurosensorial hypoacusis. This was a 4 month-old baby, who had complete KD with CAL, in whom hypoacusis was found after the resolution of the acute phase and was associated with pathological VEPs: at the following controls, VEPs were still pathological, while BAEPs were in the normal range and hearing loss had resolved. He was treated with IVIG 8 days after KD onset and did not receive steroids.

In the patients with VEP abnormalities, the prevalent recorded features were the increased latency of P100 /P2 wave-Flash VEP (P2) or the pattern VEP (P100).

There was no significant difference in incidence of evoked potentials alterations between typical or incomplete KD patients. 
TABLE 1 | BAEPs and VEPs findings (normal or pathological) were divided in three groups of KD (typical, atypical and incomplete KD).

\begin{tabular}{llllll}
\hline & Patients $\boldsymbol{n}=\mathbf{5 9}$ & $\begin{array}{l}\text { Normal BAEPs } \\
\text { and VEPs } \\
\mathbf{2 0 / 5 9}(\mathbf{3 4} \%)\end{array}$ & $\begin{array}{l}\text { Pathological } \\
\text { BAEPs only } \\
\mathbf{2 7 / 5 9}(\mathbf{4 5} \%)\end{array}$ & $\begin{array}{l}\text { Pathological } \\
\text { VEPs only 4/59 } \\
\mathbf{( 7 \% )}\end{array}$ & $\begin{array}{l}\text { Pathological } \\
\text { BAEPs and VEPs } \\
\mathbf{8 / 5 9}(\mathbf{1 4 \% )}\end{array}$ \\
\hline $\begin{array}{l}\text { Typical KD } \\
\text { Atypical KD }\end{array}$ & $37 / 59(63 \%)$ & $11 / 37(30 \%)$ & $19 / 37(51 \%)$ & $1 / 37(3 \%)$ & $6 / 37(16 \%)$ \\
Incomplete KD & $8 / 59(14 \%)$ & $4 / 8(50 \%)$ & $2 / 8(25 \%)$ & $2 / 8(25 \%)$ & 0 \\
\hline
\end{tabular}

TABLE 2 | BAEPs and VEPs findings in KD patients: 26/37 (70\%) of males and 13/22 (59\%) of females showed pathological evoked potentials.

\begin{tabular}{lllll}
\hline & $\begin{array}{l}\text { Patients with } \\
\text { pathological } \\
\text { evoked } \\
\text { potentials } \\
\boldsymbol{n}=\mathbf{3 9}\end{array}$ & $\begin{array}{l}\text { Pathological } \\
\text { BAEPs only } \\
\mathbf{2 7 / 3 9}(\mathbf{6 9} \%)\end{array}$ & $\begin{array}{l}\text { Pathological } \\
\text { VEPs only 4/39 } \\
\mathbf{( 1 0 \% )}\end{array}$ & $\begin{array}{l}\text { Pathological } \\
\text { BAEPs and VEPs } \\
\mathbf{8} / \mathbf{3 9}(\mathbf{2 1} \%)\end{array}$ \\
\hline Males (37) & $26(67 \%)$ & $18(67 \%)$ & $2(50 \%)$ & $6(75 \%)$ \\
Females (22) & $13(33 \%)$ & $9(33 \%)$ & $2(50 \%)$ & $2(25 \%)$ \\
\hline
\end{tabular}

TABLE 3 | Pathological BAEPs and VEPs and CAL: distribution of the cases.

\begin{tabular}{|c|c|c|c|c|c|}
\hline & Patients $n=59$ & $\begin{array}{l}\text { Normal BAEPs } \\
\text { and VEPs } \\
20 / 59(34 \%)\end{array}$ & $\begin{array}{l}\text { Pathological } \\
\text { BAEPs only } \\
27 / 59(46 \%)\end{array}$ & $\begin{array}{l}\text { Pathological } \\
\text { VEPs only } 4 / 59 \\
(7 \%)\end{array}$ & $\begin{array}{l}\text { Pathological } \\
\text { BAEPs and VEPs } \\
8 / 59(14 \%)\end{array}$ \\
\hline CAL & 17 (29\%) & $6 / 17$ (35\%) & 6/17 (35\%) & 1/17 (6\%) & 4/17 (24\%) \\
\hline
\end{tabular}

\section{Cardiac Involvement}

In our patients, CAL were detected in $17 / 59$ patients (29\%): six children $(10 \%)$ showed coronaritis without aneurysms, 11 (19\%) developed aneurysms. 6/17 children with CAL had normal BAEPs and VEPs; 4/17 children showed both altered BAEPs and VEPs; $6 / 17$ patients had altered BAEPs; $1 / 17$ patients had altered VEPs (Table 3).

The incidence of pathological BAEPs and VEPs was higher than CAL; in fact, $71 \%$ of the patients had normal echocardiogram, while only $34 \%$ of the patients showed normal evoked potentials. Nevertheless, 50\% of children with both pathological BAEPs and VEPs had CAL; 35\% of patients with pathological BAEPs and normal VEPs showed CAL; $6 \%$ of patients with pathological VEPs and normal BAEPs had CAL.

In some patients of our case series, evoked potentials alterations appeared earlier than CAL, while in two patients these alterations were detected only during follow-up (at 12 months after disease onset) (Table 4).

\section{Correlation With Clinical and Hematologic Parameters}

No correlation was seen between BAEPs and VEPs alterations and days of fever at the start of IVIG, pre-IVIG D-Dimer plasma levels, CRP, ESR, leukocyte count, neutrophil percentage pre- and post- IVIG, number of IVIG doses.
TABLE 4 | BAEPs and VEPs during the acute phase and 12 months after the onset of the disease.

\begin{tabular}{lll}
\hline $\begin{array}{l}\text { Patients with } \\
\text { pathological evoked } \\
\text { potentials }\end{array}$ & $\begin{array}{l}\text { Patients with } \\
\text { pathological evoked } \\
\text { potentials } \\
\text { normalized during } \\
\text { the follow-up }\end{array}$ & $\begin{array}{l}\text { Patients with normal } \\
\text { evoked potentials } \\
\text { pathological during } \\
\text { the follow-up }\end{array}$ \\
\hline 39 & 3 & 2 \\
\hline
\end{tabular}

\section{Correlation With Treatment Strategies}

Furthermore, pathological evoked potentials and the resolution of these abnormalities in 3/39 (8\%) patients during follow-up, were not correlated with corticosteroid treatment (which was administered in eight patients), while treatment with anakinra induced the resolution of CAL, as documented in the literature $(15,16)$ and the normalization of evoked potentials in one child (Figure 1).

\section{DISCUSSION}

The patients studied in our series showed altered IV, V waves and/or III-V interwave latencies, despite normal hearing acuity. These children showed a possible CNS vasculitis, and in a high percentage of them CAL were also present. 

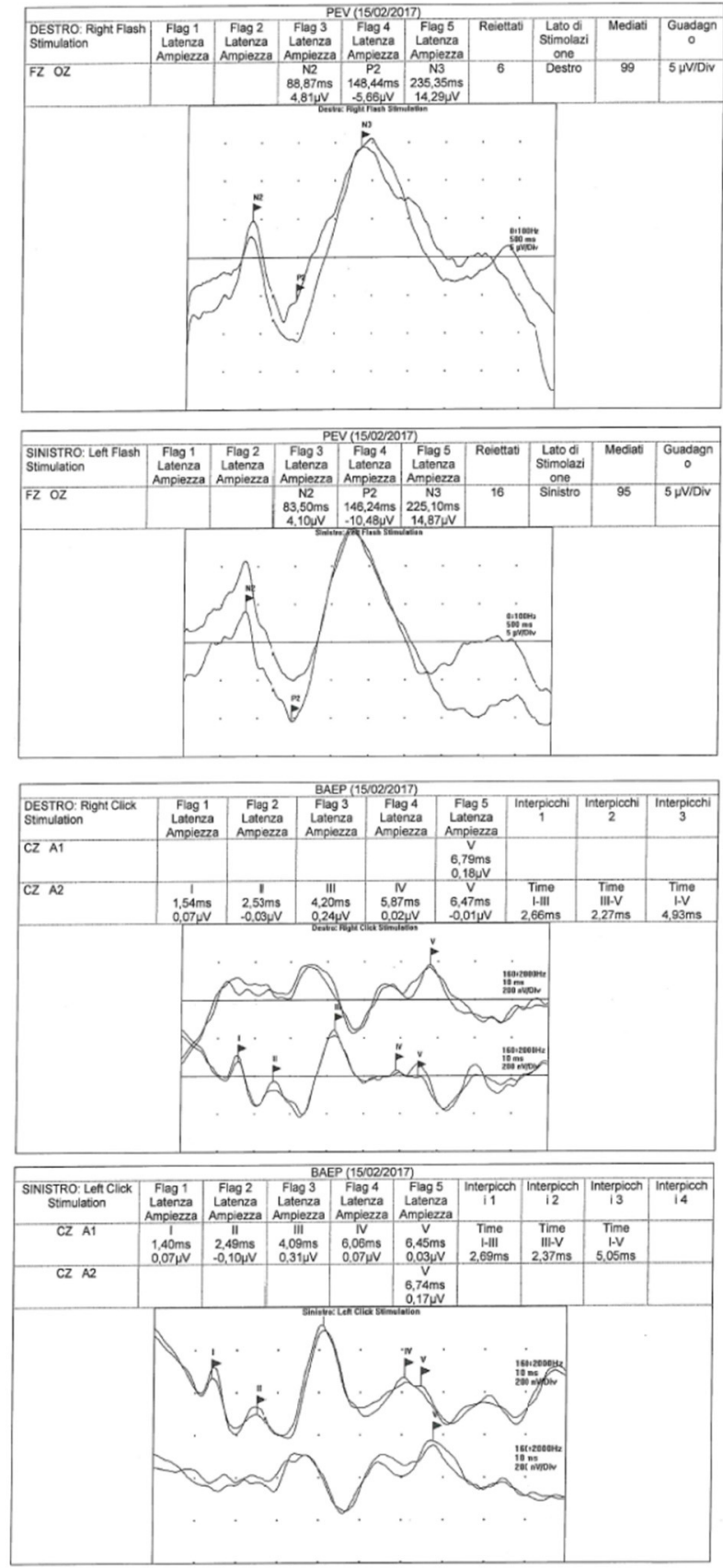
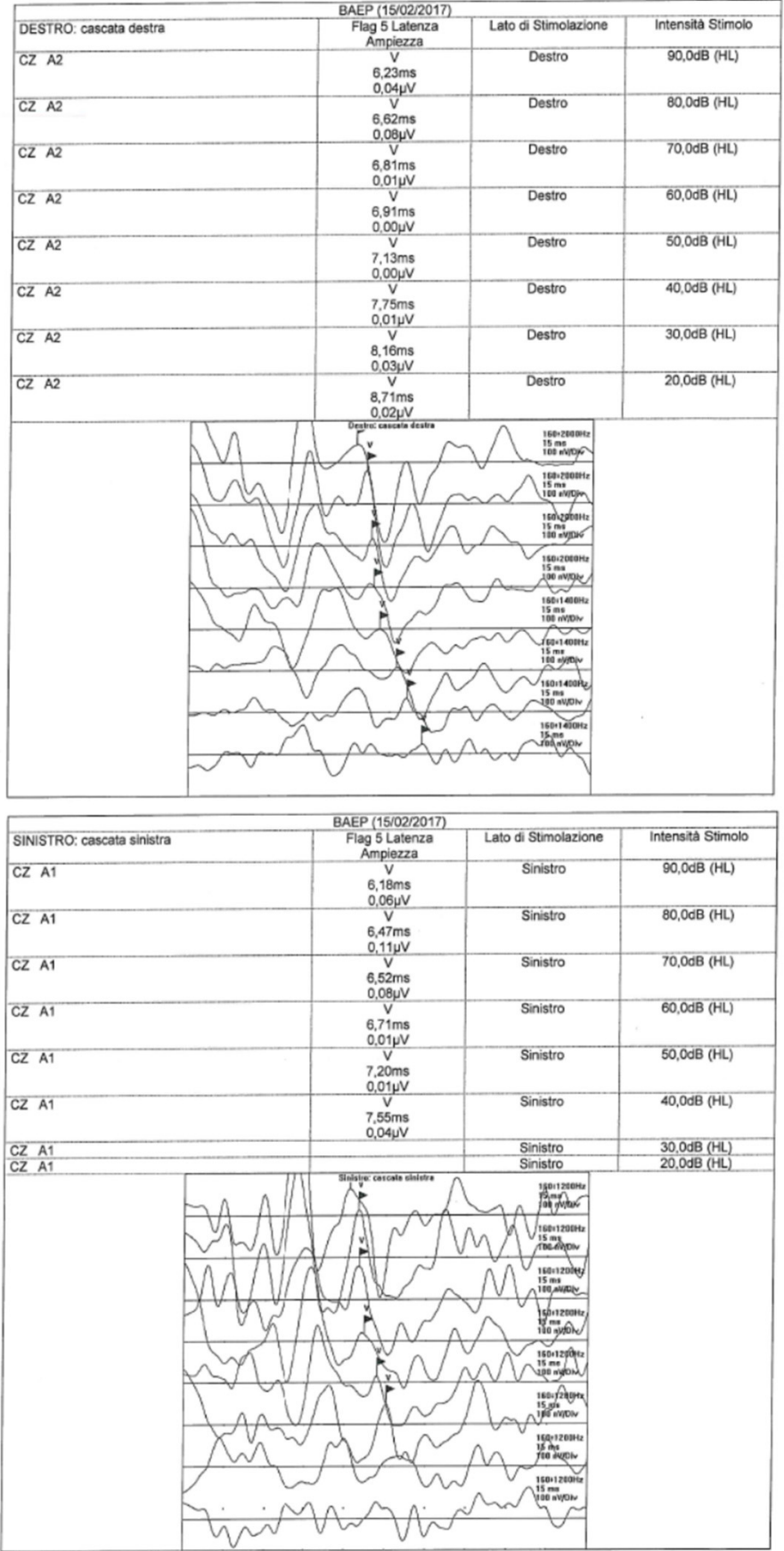

FIGURE 1 | VEPs and BAEPs in a patient with pathological records.

Recorded abnormalities of BAEPs consisted of decreased amplitude of wave V, prolonged III-V interpeak latencies caused by slowed conduction within the lower pons and the midbrain (17).

In this case series evoked potentials were pathological in a high number of patients: $70 \%$ of males and $59 \%$ of females. BAEPs, alone or in association with VEPs, were more frequently pathological than VEPs alone. In fact, isolated alteration of
VEPs was found in only $7 \%$ of the patients. Otherwise, isolated alterations of BAEPs were detected in $46 \%$ of the patients, and most of them showed alterations secondary to mesencephalic involvement.

Despite normal hearing acuity in almost all cases, the patients of our cohort showed a neuronal involvement, documented by evoked potentials. The dysfunction of our patients seemed secondary to a mesencephalic damage. This pattern could be 
an expression of a vasculitis of CNS, which has already been documented in $\mathrm{KD}$ children in other encephalic districts $(1,2,8)$. In $43 \%$ of these children CAL were present, possibly as further expression of systemic vasculitis.

The abnormalities of BAEPs and VEPs are not necessarily linked to the acute phase of $\mathrm{KD}$; in fact, these alterations have been documented also months after KD onset, and some patients may maintain a permanent loss of function with an auditory loss $(3,5,7,18-20)$. The spectrum of complications in $\mathrm{KD}$ includes not only CAL and other cardiac lesions, but also CNS involvement.

In our study, the percentage of children with pathological BAEPs and/or VEPs in association with CAL is high. However, the differences between the groups of patients with specific evoked potentials alterations did not reach the statistical significance, but the number of patients in the specific groups are small and need further confirmation in a larger population sample. Moreover, our study of CNS involvement was performed by evoked potentials, suggesting a functional involvement of the encephalic districts, but was not confirmed by Magnetic Resonance Angiography. Further studies could be integrated with Magnetic Resonance Angiography in children with $\mathrm{KD}$, to document anatomic damage secondary to systemic vasculitis.

A multicentre report on $\mathrm{KD}$ patients showed transient sensorineural hearing loss as a possible complication of acute $\mathrm{KD}$; this event was considered secondary to salicylate toxicity (21). A persistent sensorineural hearing loss is rare (19). High doses of salicylates could cause severe hearing loss; however, in our series all patients were treated with low-medium doses of ASA. Case reports described bilateral severe sensorineural hearing loss, in some cases treated with corticosteroids; some experienced only a partial improvement, with possible severe persisting hearing loss $(18,19)$.

An association between persistent sensorineural hearing loss and persistent thrombocytosis, anemia, high ESR and late administration of IVIG has been described (7); in our case series this association was not found, neither with hematological parameters nor with clinical signs. Furthermore, IVIG, steroids and ASA timing of administration was not correlated with evoked potentials alterations. Hence, the finding of evoked potentials abnormalities did not modify the treatment of our patients; however, they can help the clinicians in the diagnosis, especially in atypical or incomplete cases. In this regard, treatment can be guided by BAEPs and VEPs study, in terms of an earlier diagnostic suspicion and a prompt therapeutic choice.

A generalized vasculitis, involving CNS vessels, was documented in $\operatorname{KD}(2,22)$, describing hypoperfusion events that occur in the acute phase of $\mathrm{KD}$, and provides another

\section{REFERENCES}

1. Gitiaux C, Kossorotoff M, Bergounioux J, Adjadj E, Lesage F, Boddaert $\mathrm{N}$, et al. Cerebral vasculitis in severe Kawasaki disease: early detection by magnetic resonance imaging and good outcome after intensive physio-pathological hypothesis that supports potentials alteration. In fact, as further confirmation of the hypothesis of the systemic vasculitis, in our series several patients showed CAL as well. We could not demonstrate a statistically significant association between abnormal potential and CAL, but the limited number of children of each group did not contribute to achieve a statistically significant difference.

\section{CONCLUSION}

In conclusion, sensorineural hearing loss and alterations of evoked potentials are uncommonly reported complications of $\mathrm{KD}$. In the present sample of patients, CNS involvement was always subclinical, without symptoms otherwise reported by patients and/or parents. These data alert the clinicians about the meaning of pre-clinical evoked potentials abnormalities in the management of KD patients. We hypothesize that vasculitis of vasa nervorum is a possible pathogenetic event linked to evoked potentials alterations. Evoked potentials allow to detect brain dysfunction, supporting brain involvement and precociously helping to identify permanent lesions that can induce disability if underdiagnosed. Furthermore, the diagnosis of atypical or incomplete $\mathrm{KD}$ is further supported by evoked potentials study.

A few studies proposed that asymptomatic cerebral vasculitis might be more frequent than expected on the basis of clinical signs (23). Therefore, pediatricians must consider the possible vascular involvement beyond CAL in $\mathrm{KD}$, especially in the cerebral vessels, for the life-treating consequences and the poor prognosis of these patients.

\section{DATA AVAILABILITY STATEMENT}

The raw data supporting the conclusions of this article will be made available by the authors, without undue reservation.

\section{ETHICS STATEMENT}

The study involving human participants was reviewed and approved by the ethics committee Palermo 1 (ARNAS Civico, Palermo, Italy. The patients' legal guardian provided written informed consent to participate in this study.

\section{AUTHOR CONTRIBUTIONS}

MM, GS, and DP: substantial contributions to conception and design, acquisition of the data, analysis and interpretation of the data. RC and GC: drafted the article and revised it critically for important intellectual content. MM, GC, GS, DP, and RC: final approval of the version to be published. All named authors have agreed to its submission.

treatment. Dev Med Child Neurol. (2012) 54:1160-3. doi: 10.1111/dmcn. 12002

2. Yeom JS, Cho JY, Woo HO. Understanding the importance of cerebrovascular involvement in Kawasaki disease. Korean $J$ Pediatr. (2019). doi: 10.3345/kjp.2019.00143 
3. Aggarwal V, Etinger V, Orjuela AF. Sensorineural hearing loss in Kawasaki disease. Ann Pediatr Cardiol. (2016) 9:87-89. doi: 10.4103/0974-2069.171396

4. Novo A, Pinto S, Prior AC, Alvares S, Soares T, Guedes M. Kawasaki disease and sensorineural hearing loss: an (un)expected complication. Eur J Pediatr. (2012) 171:851-4. doi: 10.1007/s00431-011-1667-3

5. Knott PD, Orloff LA, Harris JP, Novak RE, Burns JC, Kawasaki Disease Multicenter Hearing Loss Study Group. Sensorineural hearing loss and Kawasaki disease: a prospective study. Am J Otolaryngol. (2001) 22:343-8. doi: 10.1053/ajot.2001.26495

6. Sundel RP, Cleveland SS, Beiser AS, Newburger JW, McGill T, Baker AL, et al. Audiologic profiles of children with Kawasaki disease. Am J Otol. (1992) 13:512-5.

7. Magalhães CM, Magalhães Alves NR, Oliveira KM, Silva IM, Gandolfi L, Pratesi R. Sensorineural hearing loss: an underdiagnosed complication of Kawasaki disease. J Clin Rheumatol. (2010) 16:322-5. doi: 10.1097/RHU.0b013e3181f603bc

8. Alves NR, Magalhães CM, Almeida Rde F, Santos RC, Gandolfi L, Pratesi R. Prospective study of Kawasaki disease complications: review of 115 cases. Rev Assoc Med Bras. (2011) 57:295-300. doi: 10.1016/S2255-4823(11)70062-5

9. Newburger JW, Takahashi M, Gerber MA, Gewitz MH, Tani LY, Burns JC, et al.. Diagnosis, treatment, and long-term management of Kawasaki disease: a statement for health professionals from the Committee on Rheumatic Fever, Endocarditis and Kawasaki Disease, Council on Cardiovascular Disease in the Young, American Heart Association. Circulation. (2004) 110:2747-71. doi: 10.1161/01.CIR.0000145143.19711.78

10. Marchesi A, Tarissi de Jacobis I, Rigante D, Rimini A, Malorni W, Corsello G, et al. Kawasaki disease: guidelines of the Italian society of pediatrics, part I-definition, epidemiology, etiopathogenesis, clinical expression and management of the acute phase. Ital J Pediatr. (2018) 44:102. doi: 10.1186/s13052-018-0536-3

11. Marchesi A, Tarissi de Jacobis I, Rigante D, Rimini A, Malorni W, Corsello G, et al. Kawasaki disease: guidelines of the Italian society of pediatrics, part II-treatment of resistant forms and cardiovascular complications, follow-up, lifestyle and prevention of cardiovascular risks. Ital J Pediatr. (2018) 44:103. doi: 10.1186/s13052-0180529-2

12. Celesia GG, Bodis-Wollner I, Chatrian GE, Harding GF, Sokol S, Spekreijse H. Recommended standards for electroretinograms and visual evoked potentials. Report of an IFCN committee. Electroencephalogr Clin Neurophysiol. (1993) 87:421-36. doi: 10.1016/0013-4694(93)90157-Q

13. Holder GE, Celesia GG, Miyake Y, Tobimatsu S, Weleber RG, International Federation of Clinical Neurophysiology. International Federation of Clinical Neurophysiology: recommendations for visual system testing.
Clin Neurophysiol. (2010) 121:1393-409. doi: 10.1016/j.clinph.2010. 04.010

14. American Clinical Neurophysiology Society. Guideline 9C: Guidelines on short-latency auditory evoked potentials. J Clin Neurophysiol. (2006) 23:157-67. doi: 10.1097/00004691-200604000-00012

15. Kone-Paut I, Cimaz R, Herberg J, Bates O, Carbasse A, Saulnier $\mathrm{JP}$, et al. The use of interleukin 1 receptor antagonist (anakinra) in Kawasaki disease: a retrospective cases series. Autoimmun Rev. (2018) 17:768-74. doi: 10.1016/j.autrev.2018.01.024

16. Maggio MC, Cimaz R, Alaimo A, Comparato C, Di Lisi D, Corsello G. Kawasaki disease triggered by parvovirus infection: an atypical case report of two siblings. J Med Case Rep. (2019) 13:104. doi: 10.1186/s13256-019-2028-5

17. Zaher A. Visual and brainstem auditory evoked potentials in neurology. In: Schwartz M, editor. EMG Methods for Evaluating Muscle and Nerve Function. London: IntechOpen (2012). p. 281-310. doi: 10.5772/26375

18. Kim KY, Kim KH, Park YA, Seo YJ. Kawasaki disease and labyrinthitis: an underdiagnosed complication. J Audiol Otol. (2017) 21:53-56. doi: 10.7874/jao.2017.21.1.53

19. Smith KA, Yunker WK. Kawasaki disease is associated with sensorineural hearing loss: a systematic review. Int J Pediatr Otorhinolaryngol. (2014) 78:1216-20. doi: 10.1016/j.ijporl.2014.05.026

20. Suzuki H, Yanagawa T, Kihira S. Two cases of hearing loss associated with kawasaki disease. Clin Pediatr. (1988) 41:167-72.

21. Kim SM, Jo JM, Baek MJ, Jung KH. A case of bilateral sudden hearing loss and tinnitus after salicylate intoxication. Korean J Audiol. (2013) 17:23-6. doi: 10.7874/kja.2013.17.1.23

22. Amano S, Hazama F. Neutral involvement in kawasaki disease. Acta Pathol Jpn. (1980) 30:365-73. doi: 10.1111/j.1440-1827.1980.tb01331.x

23. Ichiyama $T$, Nishikawa M, Hayashi T, Koga M, Tashiro N, Furukawa S. Cerebral hypoperfusion during acute Kawasaki disease. Stroke. (1998) 29:1320-1. doi: 10.1161/01.STR.29.7.1320

Conflict of Interest: The authors declare that the research was conducted in the absence of any commercial or financial relationships that could be construed as a potential conflict of interest.

Copyright (C) 2020 Maggio, Corsello, Salvo, Puma and Cimaz. This is an open-access article distributed under the terms of the Creative Commons Attribution License (CC $B Y)$. The use, distribution or reproduction in other forums is permitted, provided the original author(s) and the copyright owner(s) are credited and that the original publication in this journal is cited, in accordance with accepted academic practice. No use, distribution or reproduction is permitted which does not comply with these terms. 